\title{
Evidence Based Practice and Evidence based Nursing Education
}

\author{
Dimitrios Theofanidis*
}

Nursing Department, Alexandreio Tehnological Educational Institute of Thessaloniki, Greece

*Corresponding author: Dimitrios Theofanidis, Clinical Professor, Nursing Department, Alexandreio Tehnological Educational Institute of Thessaloniki, Greece, Tel: +30 6945227796, E-mail: dimitrisnoni@yahoo.gr

Received date: June 13, 2015; Accepted date: July 02, 2015; Published date: Jul 10, 2015

Copyright: (c) 2015 Theofanidis D. This is an open-access article distributed under the terms of the Creative Commons Attribution License, which permits unrestricted use, distribution, and reproduction in any medium, provided the original author and source are credited.

\section{Introduction}

Since the beginning of the new millennium, professional nursing practice has evolved from Research Utilization (RU) to Evidence-Based Practice (EBP) mainly in sophisticated care delivery systems. EBP requires integration of best research evidence coupled with clinical expertise, patient values and needs within a conscientious costeffective health care delivery. Moreover, it can provide a solid and supportive framework for clinical practice that integrates the best available scientific evidence with the expertise of the clinician and with patients' preferences and values to make sound decisions about health care [1].

The aim of this short commentary is to describe critically the merits of integrating EBP in contemporary nursing education. Also, this discussion paper will cite aims and objectives of implementing EBP, present a literature review of the main activities in teaching EBP and suggest approaches to inform nursing students on optimum healthcare outputs.

\section{Discussion}

With regards to nursing education, the teaching of Evidence Based Nursing requires updated knowledge of EBP and reflection in one's role as nurse teacher. In this sense, and by paraphrasing Sackett's famous definition: EBP in teaching nurses requires the conscientious, explicit and judicious use of current best research findings in making decisions about the performance of one's role as a teacher and the learning needs of his/her students. EBP also ensures that nursing delivery remains constantly concerned with mistakes and shortcomings in everyday clinical nursing care. In this light, the undergraduate teaching of Evidence Based Nursing (EBN) is the basic 'educational springboard' for future staff nurses to be able to initiate improvements and change of practice [2,3].

The aims and objectives of implementing EBP in undergraduate nurses are as follows:

- To teach students the art and science of updating their practice evidence base throughout their professional careers.

- To focus on pragmatic economical ways of improving health outcomes in their routine practice.

- To stimulate an interest in nurses to partake actively in clinical decision making.

- To learn to create a stimulating learning environment for other staff and nursing students.

- To be able to uncover EBP solutions in order to improve nursing care.

- To encourage constructive change according to EBP principles.
Preliminary EBP in nursing education started off using general recomendations from research papers, that is research utilization per se. Later on, this ethos evolved via reviews and meta-analysis providing evidence based findings which formed the foundations of current optimum practices. It should be noted that basic research utilization is still prevalent across many countries in Europe and beyond. This is eronously thought to be synonymous with EBP. Yet, nurse educators should be able to recognize that research utilization is only a basic step towards achieving evidence based practice status. On the other hand, nursing students need to be taught about differences and similarities in some terminologies and thus be able to differentiate between: recommendations, guidelines, care pathways and clinical protocols and EBP.

There is a variety of grading systems for evidence and recommendations currently in use as proposed by bodies and associations such as the American Heart Association (USA), the National Institute for Health and Clinical Evidence (UK), the Cochrane Collaboration (UK), the Joanna Briggs Institute (Australia) and more recently, the Guideline Recommendation and Evidence Grading (UK). However, it should be noted that these were originally developed as clinical governance tools rather than educational resources. Moreover, there have been criticisms about the number of similar evidence systems currently in use and that many are primarily related to the evidence in the literature and not always to clinical significance $[4,5]$.

Hence, the validity of any effort to teach EBP would depend on the type and level of evidence on which they are based. Furthermore, nursing needs mainly to evaluate the validity and then reliability of the contents of such educational outcomes and achievements and should be incorporated into the teaching agendas of nursing educational institutions which should improve their quality of teaching, with improved nursing care delivery.

These state-of-the-art professional abilities could be transferred to any health care system within the European Union and beyond.

Main activities in teaching EBP to nurse undergraduates include teaching on:

- Framing a good clinical question,

- Ways of finding the evidence,

- Critical skills in assessing the evidence,

- Enabling students to make wise and informed clinical decisions.

It should be noted that in any attempt to teach EBP, students should be offered state- of-the-art e-learning facilities and access to free use of evidence based resources such as the Cochrane Collaboration, NICE and the Joanna Briggs Institute. 
Citation: Theofanidis D (2015) Evidence Based Practice and Evidence based Nursing Education . J Nurs Care 4: 279. doi: 10.4172/2167-1168.1000279

Page 2 of 2

Although most nursing schools place increasing importance on the teaching of subject areas related to EBP, such as research methods, many curricula do not give practical strategic advice on how to implement EBP in the context of routine practice. The innovative aspect of such teaching should entail practical examples and proposed change of practice. Yet, such an enthusiastic endeavour should also include differences and similarities when interpreting the strength of evidence [6].

When staff broadens their teaching of EBP, in effect, student nurses are enlightened on how to approach EBP as part of their future vocation. On completion of such an educational intervention, students should have attained the following skills, i.e. be able to:

- Identify areas that require improvement in their nursing practice environment,

- Describe the lack of evidence in routine clinical tasks,

- Discuss critically the rigor of the evidence found,

- Discuss critically gaps of evidence in routine nursing practice.

- Explain implications for nursing practice.

- Foster the skill of interpreting EBP as a means of stimulating the creation of pragmatic solutions to current health problems within Europe.

- Produce a straightforward summary of simple, cost effective solutions as opposed to suggesting the implementation of unrealistic and expensive solutions in the present difficult financial circumstances confronting many European countries.

Furthermore, the educational impact of EBP teaching goes beyond clinical nursing per se as it also provides or furthers skills for students and staff alike, in structuring information and improving decision making by using rigorous evidence. This is useful throughout life in their chosen professions. Also, by being able to develop critical thinking skills in appraising evidence as presented to them, both staff and students will learn that cross-topic stimulation can create unusual ways of problem solving $[7,8]$.

\section{Conclusions}

The present widespread financial recession and its global implications have coincidentally hit many countries hard, and there is increasing concern about cuts in healthcare budgeting. The steady increase of migration and poverty has put an additional strain on national health care systems throughout Europe and beyond. Therefore, health care providers now need to be more effective with relatively fewer resources. However, in such adverse working climates, it is still necessary for nurses to remain updated and also to be able to find, assess and implement best evidence in their everyday care also considering vocational, financial and geopolitical restrictions.

Thus, by being taught EBP, a student nurse would learn to explore the key concepts within the scientific paradigm of identifying, appraising and using research evidence in order to find innovative ways of improving nursing care. However, this teaching should follow the principles of critical thinking, which includes that a student is skeptical about research findings per se and contemplates the interpretation of results in realistic terms and relevant circumstances.

\section{References}

1. Fink R, Thompson CJ, Bonnes D (2005) Overcoming barriers and promoting the use of research in practice. J Nurs Adm 35: 121-129.

2. Sackett DL, Rosenberg WM, Gray JA, Haynes RB, Richardson WS (1996) Evidence based medicine: what it is and what it isn't. BMJ 312: 71-72.

3. Brownson R, Colditz G, Proctor E. (2012). Dissemination and implementation research in health: Translating science to practice, Oxford University Press, Inc, New York.

4. Harbour R, Miller J (2001) A new system for grading recommendations in evidence based guidelines. BMJ 323: 334-336.

5. Smith F, Tong J, Smith J (2006) Evidence-based medicine. Contin Educ Anaesth Crit Care Pain. 6: 148-151.

6. Moch SD, Cronje RJ, Branson J (2010) Part 1. Undergraduate nursing evidence-based practice education: envisioning the role of students. J Prof Nurs 26: 5-13.

7. Cronje R, Moch S (2010) Part III. Reenvisioning undergraduate nursing students as opinion leaders to diffuse evidence-based practice in clinical settings. J Prof Nurs. 26: 23-28.

8. Emerson RJ, Records K (2008) Today's challenge, tomorrow's excellence: the practice of evidence-based education. J Nurs Educ 47: 359-370. 